\title{
Dummy Molecularly Imprinted Polymers-Capped CdTe Quantum Dots for the Fluorescent Sensing of 2,4,6-Trinitrotoluene
}

\author{
Shoufang Xu, ${ }^{\dagger, \ddagger}$ Hongzhi Lu, ${ }^{\dagger}$ Jinhua Li, ${ }^{\dagger}$ Xingliang Song, ${ }^{\dagger}$ Aixiang Wang, ${ }^{\dagger}$ Lingxin Chen, ${ }^{*} \neq$ \\ and Shaobo $\mathrm{Han}^{\dagger}$ \\ ${ }^{\dagger}$ School of Chemistry \& Chemical Engineering, Linyi University, Linyi 276005, China \\ ${ }^{\ddagger}$ Key Laboratory of Coastal Zone Environmental Processes and Ecological Remediation, Yantai Institute of Coastal Zone Research, \\ Chinese Academy of Sciences, Yantai 264003, China \\ ${ }^{\S}$ University of Chinese Academy of Sciences, Beijing 100049, China
}

\section{Supporting Information}

ABSTRACT: Molecularly imprinted polymers (MIPs) with trinitrophenol (TNP) as a dummy template molecule capped with CdTe quantum dots (QDs) were prepared using 3aminopropyltriethoxy silane (APTES) as the functional monomer and tetraethoxysilane (TEOS) as the cross linker through a seedgrowth method via a sol-gel process (i.e., DMIP@QDs) for the sensing of 2,4,6-trinitrotoluene (TNT) on the basis of electrontransfer-induced fluorescence quenching. With the presence and increase of TNT in sample solutions, a Meisenheimer complex was formed between TNT and the primary amino groups on the surface of the QDs. The energy of the QDs was transferred to the complex, resulting in the quenching of the QDs and thus decreasing the fluorescence intensity, which allowed the TNT to

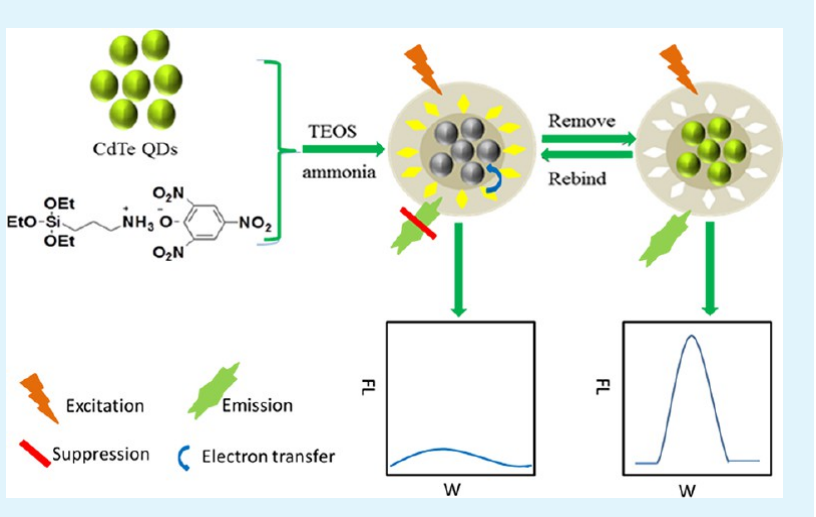
be sensed optically. DMIP@QDs generated a significantly reduced fluorescent intensity within less than $10 \mathrm{~min}$ upon binding TNT. The fluorescence-quenching fractions of the sensor presented a satisfactory linearity with TNT concentrations in the range of $0.8-30 \mu \mathrm{M}$, and its limit of detection could reach $0.28 \mu \mathrm{M}$. The sensor exhibited distinguished selectivity and a high binding affinity to TNT over its possibly competing molecules of 2,4dinitrophenol (DNP), 4-nitrophenol (4-NP), phenol, and dinitrotoluene (DNT) because there are more nitro groups in TNT and therefore a stronger electron-withdrawing ability and because it has a high similarity in shape and volume to TNP. The sensor was successfully applied to determine the amount of TNT in soil samples, and the average recoveries of TNT at three spiking levels ranged from 90.3 to $97.8 \%$ with relative standard deviations below $5.12 \%$. The results provided an effective way to develop sensors for the rapid recognition and determination of hazardous materials from complex matrices.

KEYWORDS: quantum dots, molecularly imprinted polymers, dummy template, fluorescent detection, seed-growth method, 2,4,6-trinitrotoluene

\section{INTRODUCTION}

Over the past decade, semiconductor quantum dots (QDs) have attracted intensive research interest for sensing and recognizing the organic and inorganic compounds in challenging environments because of their prominent advantages, such as great photostability, high photoluminescence efficiency, size-dependent emission wavelengths, and sharp emission profile. A variety of QD-based sensors have been reported for ions, ${ }^{1}$ biomacromolecules, ${ }^{2-4}$ and small organic molecules. ${ }^{5}$ To improve further the selectivity of QD-based sensors, a molecular-imprinting technique has been introduced to tailor the selectivity of analytes by the polymer materials (i.e., molecularly imprinted polymers (MIPs)). ${ }^{6}$ MIPs are prepared by the copolymerization of functional monomers and cross linkers in the presence of target analytes, which act as template molecules. $^{6-9}$ After the removal of the template, recognition sites complementary in size, shape, and functionality to the template are formed in the 3D polymer network. ${ }^{6,10,11}$ Recently, MIPs have aroused extensive attention and been widely applied in many fields, such as extraction/separation $^{12-15}$ and chemo/biosensing, ${ }^{16-18}$ owing to their desired selectivity, physical robustness, thermal stability, low cost, and easy preparation. Up until now, a number of MIPs-capped QD sensors that combine the selectivity of MIPs with the sensitivity of QDs have been developed to detect various compounds such as pentachlorophenol, ${ }^{19}$ 4-nitrophenol, ${ }^{20}$ pyrethroids, ${ }^{21}$ proteins, $^{22,23}$ and domoic acid. ${ }^{24}$ Inspired by these studies, we expect to construct a new MIPs-capped QD sensor that can

Received: June 7, 2013

Accepted: July 22, 2013

Published: July 22, 2013 
recognize and bind explosives, such as 2,4,6-trinitrotoluene (TNT).

TNT, one of the powerful explosive nitroaromatic compounds, has raised worldwide concerns regarding public security and environmental problems because of its wide production and use by the military and in engineering. In the recent years, many chemosensors have been developed for the real-time detection of nitroaromatics. ${ }^{25-31}$ For example, Xia et al. designed a fluorescence resonance energy-transfer system consisting of a gold nanorod and QDs for the turn-on fluorescent sensing of TNT in the near-infrared region. ${ }^{27}$ Gao et al. reported a resonance energy-transfer-amplifying fluorescence quenching at the surface of silica nanoparticles for the ultrasensitive detection of TNT. ${ }^{28}$ Stringer et al. prepared a fluorescent QDs-labeled imprinted polymer for the detection of nitroaromatic explosives in solution. ${ }^{30}$ Instead of preparing the QDs beforehand and incorporating them into the imprinted polymers, QDs were added as a postprocessing step. ${ }^{30}$ By combining the fluorescence-quenching transduction mechanism with the amplifying feature of conjugated polymers, several chemical sensors on the basis of amplifying fluorescentconjugated polymers for TNT have been developed, which is well reviewed by T. M. Swager. ${ }^{31}$ However, to the best of our knowledge, MIPs-capped QDs for TNT have not been reported.

Meanwhile, it is well known that TNT invloves safety concerns and cannot be obtained easily. Accordingly, a dummy molecular-imprinting technique could be employed using structural analogs of the targeted compounds as template molecules. This technique has been considered to be more effective to prepare MIPs to solve the problem of template leakage $e^{32,33}$ as well as offering an attractive alternative under the following conditions: (1) when the original template is very expensive or involves safety considerations for its manipulation ${ }^{6}$ and (2) when the condition used to polymerize could result in unwanted compound degradation or the low solubility of the targeted analytes does not allow its use for the synthesis of MIPs. ${ }^{6}$

Herein, trinitrophenol (TNP), whose structure is similar to that of TNT, was chosen as a dummy template molecule, and 3-aminopropyltriethoxysilane (APTES) and tetramethoxysilane (TEOS) were selected as the functional monomer and cross linker, respectively, for the preparation of a high-affinity DMIP@QDs by a sol-gel process for the recognition and sensing of trace TNT in soil samples. The developed DMIP@ QDs could highly selectively rebind TNT and quickly quench the fluorescence of the QDs and therefore is capable of accurate quantification in a broad linear range. Moreover, this sensor had economic and eco-friendly advantages over chromatographic methods. Furthermore, the sensing strategy might provide an attractive alternative to rapidly monitor various targets by virtue of a electron-transfer-induced fluorescence quenching mechanism.

\section{EXPERIMENTAL SECTION}

Reagents. Tellurium powder, $\mathrm{Cd}\left(\mathrm{NO}_{3}\right)_{2} \cdot 4 \mathrm{H}_{2} \mathrm{O}$, thioglycollic acid (TGA), sodium borohydride, tetraethyl orthosilicate (TEOS), trinitrophenol (TNP), cyclohexane, $n$-hexanol, and Triton X-100 (TX-100) were purchased from Tianjin Reagent Plant (Tianjin, China). Fluorescamine and 3-aminopropyl triethoxy silane (APTES) were purchased from J\&K Technology Ltd. (Beijng, China). Poly(dimethyldiallyl ammonium chloride) (PDDA, $\left.M_{\mathrm{w}}=70000\right)$, TNT standard solution (10 mg/L), dinitrotoluene (DNT), 2,4dinitrophenol (DNP), 4-nitrophenol (4-NP), and phenol were purchased from Sigma-Aldrich (Shanghai, China). All other affiliated reagents and materials were all supplied by Sinopharm Chemical Reagent Co. Ltd. (Shanghai, China). All reagents were of analytical grade and used without further purification unless otherwise specified. Aqueous solutions were prepared with freshly deionized water (18.2 $\mathrm{M} \Omega$ specific resistance) obtained with a Pall Cascada laboratory water system (Millipore, Bedford, MA, USA).

Characterization. Fluorescence measurements were performed with a Fluoromax-4 Spectrofluorometer (Horiba Scientific) equipped with $1 \mathrm{~cm}$ quartz cell at $25{ }^{\circ} \mathrm{C}$, with excitation and emission slit widths of 6 and $6 \mathrm{~nm}$, respectively, and the excitation wavelength at $380 \mathrm{~nm}$. The morphological evaluation was examined with a scanning electron microscope (SEM, Hitachi S-4800 FE-SEM, operating at $5 \mathrm{kV}$ ) and a transmission electron microscope (TEM, JEM-2100F). The HPLCUV (Skyray Instrument Inc., China) conditions employed for TNT separation and determination were as follows: a $\mathrm{C}_{18}$ column with 250 $\times 4.6 \mathrm{~mm}^{2}$ i.d. (Arcus EP-C ${ }_{18}, 5 \mu \mathrm{m}$, Waters, USA) was used as the analytical column, methanol/water $(50: 50, \mathrm{v} / \mathrm{v})$ was used as the

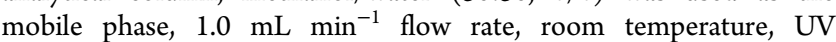
detection at $254 \mathrm{~nm}$, and an injection volume of $10 \mu \mathrm{L}$. Molecular structures were optimized with Gaussian 03 using B3LYP/6-31G**.

Synthesis of Water-Soluble CdTe QDs. Water-soluble TGAmodified CdTe QDs were synthesized according to the reported method $^{34}$ with the necessary modification of TGA to replace mercaptosuccinic acid. Briefly, $92.4 \mathrm{mg}$ of $\mathrm{Cd}\left(\mathrm{NO}_{3}\right)_{2} \cdot 4 \mathrm{H}_{2} \mathrm{O}, 63 \mu \mathrm{L}$ of TGA, and $75 \mathrm{~mL}$ of distilled water were mixed in a three-necked flask to form the cadmium precursor. The mixture was adjusted to $\mathrm{pH}$ 9 to 10 with $1.0 \mathrm{M} \mathrm{NaOH}$ and stirred under $\mathrm{N}_{2}$ for $30 \mathrm{~min}$. Next, 1 $\mathrm{mL}$ of freshly prepared NaHTe aqueous solution(using $40 \mathrm{mg}$ of $\mathrm{NaBH}_{4}$ and $38.3 \mathrm{mg}$ of tellurium powder) was injected into the reaction system under stirring. The solution was heated until boiling and refluxed for $2 \mathrm{~h}$. The resultant TGA-capped CdTe QDs exhibited strong kelly-green fluorescence at $530 \mathrm{~nm}$.

Preparation of MIPs-Capped QDs. Molecularly imprinted silica nanospheres with embedded CdTe QDs are usually prepared by two methods (i.e., the reverse-microemulsion and Stöber methods). For the typical reverse-microemulsion method, ${ }^{34} 1.8 \mathrm{~mL}$ of TX-100 and $1.8 \mathrm{~mL}$ of $n$-hexanol were dispersed in $7.5 \mathrm{~mL}$ of cyclohexane, $500 \mu \mathrm{L}$ of TGA-capped CdTe QDs, $60 \mu \mathrm{L}$ of PDDA solution $(0.075 \%$, v/v), and $60 \mu \mathrm{L}$ of ammonia to form a microemulsion. Next, $100 \mu \mathrm{L}$ of TEOS was added to the microemulsion system to initiate the hydrolysis. After the mixture was stirred for $5 \mathrm{~h}, 20 \mu \mathrm{L}$ of APTES and $5 \mathrm{mg}$ of TNP in $0.2 \mathrm{~mL}$ cyclohexane were added, and the mixture was stirred for $24 \mathrm{~h}$. Finally, the microemulsion was broken by $10 \mathrm{~mL}$ of acetone, and the resultant precipitate was washed in sequence with ethanol and water. For typical Stöber method, ${ }^{35}$ a $20 \mathrm{~mL}$ solution of $100 \mathrm{mg}$ of TNP in anhydrous ethanol and $250 \mu \mathrm{L}$ of APTES were added to a $50 \mathrm{~mL}$ flask. After stirring for $1 \mathrm{~h}, 1.0 \mathrm{~mL}$ of TEOS, $5 \mathrm{~mL}$ of CdTe QDs, and $1.0 \mathrm{~mL}$ of ammonia were added to the above mixture and kept stirring for $24 \mathrm{~h}$. Meanwhile, the seed-growth method was also used to prepare the MIPs-capped QDs, and the typical process was as follows. First the "seed" (MIP@QDs with diameter of about 50 $\mathrm{nm}$ ) was prepared by the reverse-microemulsion method as described above. Next, $50 \mathrm{mg}$ of the seed was added into $20 \mathrm{~mL}$ of an anhydrous ethanol solution that contained $100 \mathrm{mg}$ of TNP, $250 \mu \mathrm{L}$ of APTES, 5 $\mathrm{mL}$ of CdTe QDs, and $1.0 \mathrm{~mL}$ of ammonia. After stirring for $1 \mathrm{~h}, 1.0$ $\mathrm{mL}$ of TEOS was added dropwise to the above mixture and kept stirring for $24 \mathrm{~h}$. The obtained MIPs-coated QDs were centrifuged and washed to remove the original templates in the imprinted silica nanoparticles with a mixture solvent of ethanol/acetonitrile (8:2) as reported, ${ }^{21}$ which was repeated several times until no template was detected by UV-vis spectrophotometry. For the three methods, the nonimprinted polymers (NIPs)-capped QDs were prepared in the same manner but without adding template molecules.

Analysis of Soil Samples. Soil samples were used to demonstrate the applicability of the MIPs-capped QDs for the detection of TNT. Soil samples were randomly collected from farm land located in the suburbs of Yantai City, and an acetone-based extraction method was used to retrieve TNT from the soil samples as reported. ${ }^{36}$ Briefly, extracts were prepared by mixing $2.0 \mathrm{~g}$ of dry and well-homogenized 
soil samples with $10 \mathrm{~mL}$ of acetone in glass vials. The samples were shaken for $1 \mathrm{~h}$ and filtered through a $0.45 \mu \mathrm{m}$ syringe filter. The acetone extracts were stored in the dark at $-20{ }^{\circ} \mathrm{C}$ for use. Immediately prior to analysis, $2 \mathrm{~mL}$ of the acetone extracts was transferred to glass test tubes, and the acetone was evaporated by a brief treatment with a stream of nitrogen followed by dissolution in 0.5 $\mathrm{mL}$ of ethanol. The TNT concentration in each of these samples was determined by the QDs fluorescence quenching.

\section{RESULTS AND DISCUSSION}

Preparation and Characterization of DMIP@QDs. Considering that TNT involves safety considerations and cannot be obtained easily, a dummy molecular-imprinting technique was used to prepare MIPs for the recognition of TNT. TNP has a similar shape and volume to TNT, as seen from Figures 1A and S1, and more importantly it is relatively

(A)
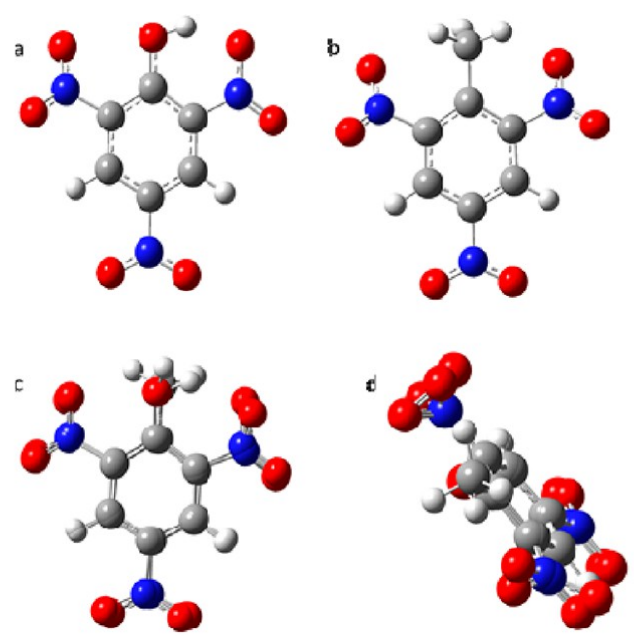

(B)

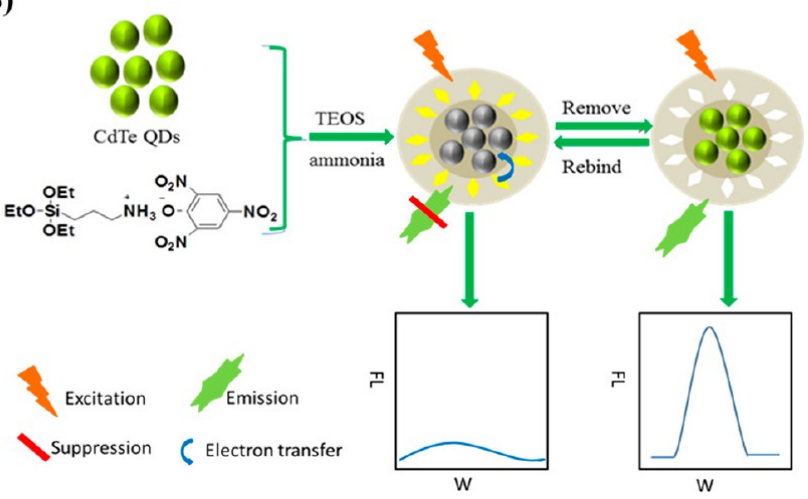

Figure 1. (A) Molecular modeling of TNP (a) and TNT (b) and the superposition of TNT (c) and TNP (d). Structures were optimized with Gaussian 03 using B3LYP/6-31G**. (B) Schematic illustration for the preparation of DMIP@QDs and the sensing mechanism for TNT.

stable/safe and can be obatined freely; therefore, TNP as the TNT analogue was used as the template molecule to prepare MIPs for the determination of TNT. The dummy MIPs-capped CdTe QDs (DMIP@QDs) were prepared by a sol-gel process, and the major steps involved in the imprinting synthesis are shown in Figure 1B. The silica nanospheres were simply fabricated by means of the hydrolysis and condensation reaction of APTES and TEOS in the presence of aqueous ammonia solution as the catalyst.

There are two methods commonly used to prepare silica coated QDs, the reverse-microemulsion and Stöber methods. In Figure 2, the SEM and TEM images and fluorescence spectra of the nanoparticles prepared by the different methods are shown. Figure 2A shows that the DMIP@QDs prepared by the revere-microemulsion method (DMIP-R) had a highly uniform spherical morphology with diameter of about $50 \mathrm{~nm}$, and QDs were embedded into the silica nanoparticles. However, the shape of the fluorescence spectrum for DMIP-R was unsatisfactory, as can be seen from Figure 2D curve a. The fairly wide peak revealed that the fluorescence intensity was not sensitive to the change of the TNT concentration (Figure S2A). The shape of the fluorescence spectra for DMIPs@QDs prepared by the Stöber method (DMIP-S) was similar to that of the QDs, as seen from Figure 2D curves $b$ and $d$, respectively. Unfortunately, the morphology was undesirable. As shown in Figure 2B, some little particles with a diameter of about $50 \mathrm{~nm}$ aggregated into irregular big particles, which was easily sedimented in solution. In addition, the large volume of the DMIP-S particles was unfavorable for the sensitive detection of TNT (Figure S2B).

Consequently, DMIP@QDs with an ideal morphology and fluorescence spectrum were expected to be prepare by the seedgrowth method, which combined the advantages of the abovementioned two methods. The seed was first prepared by the reverse-microemulsion method, and the final DMIP@QDs particles were prepared by the Stöber method. As far as we know, this is the first time that DMIP@QDs were prepared by the seed-growth method. As seen, the attained DMIP@QDs particles were highly spherical in shape and uniform in size (Figure 2C) and possessed a favorable fluorescence spectrum (Figure 2D curve c) simultaneously. The regular morphology and smaller particle size provided strong support for improving the detection sensitivity of DMIP@QDs. By considering the morphology, fluorescence spectrum, and detection sensitivity comprehensively, the seed-growth method was adopted to prepare DMIP@QDs for TNT sensing using TNP as the template, APTES as the functional monomer, and TEOS as the cross linker.

Besides being characterized by SEM and TEM, the fluorescence property of DMIP@QDs was also examined. Figure $3 \mathrm{~A}$ shows the fluorescence spectra of QDs and MIPs or NIPs-capped QDs. The spectrum of DMIP@QDs showed a slight red shift (Figure $3 \mathrm{~A}$ curve $\mathrm{b}$ ), and the fluorescence intensity partly decreased during the preparation process in comparison with that of the QDs (Figure 3A curve a). This is consistent with a previous report ${ }^{37}$ (i.e., a single charge close to the QDs surface could generate an electric field), which was sufficiently large to cause fluorescence quenching and a red shift. The fluorescence stability was evaluated by the repeated detection of the fluorescence emission intensity every $10 \mathrm{~min}$. The result shown in Figure 3B indicated a stable emission of QDs within $90 \mathrm{~min}$. Moreover, the fluorescence intensity change during the storage of DMIP@QDs was also investigated. The repeated detection of the fluorescence emission intensity was performed every day. As seen in Figure S3, when the sensor was stored for 7 days, the intensity retained $96 \%$ of its initial response. This result implies that the developed fluorescence sensor has acceptable storage stability. The maintained fluorescence intensity within $90 \mathrm{~min}$ and 1 

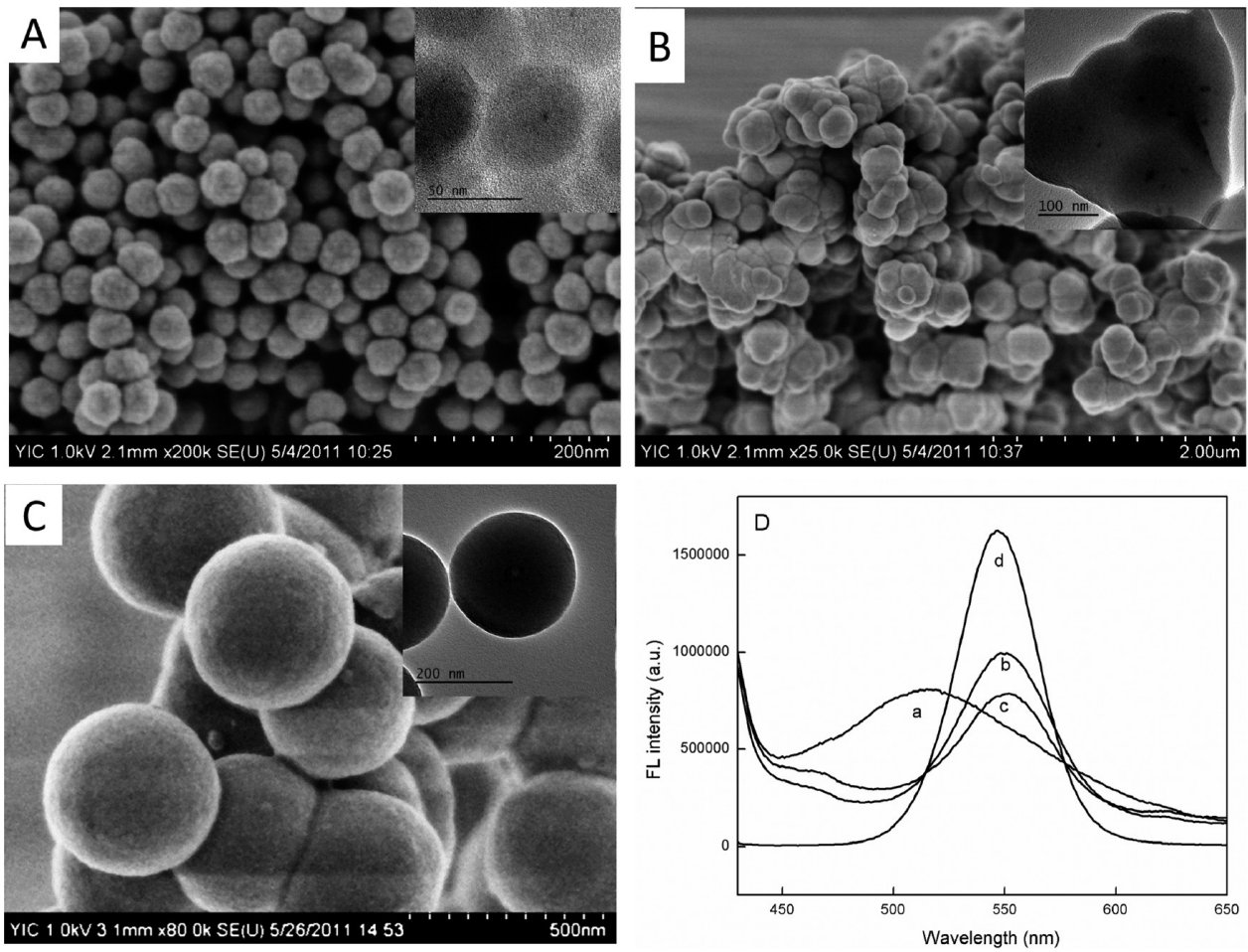

Figure 2. SEM and TEM (insets) images of DMIP@QDs prepared by (A) the reverse-microemulsion method, (B) the Stöber method, and (C) the seed-growth method. (D) Fluorescence spectra of DMIP@QDs prepared by (a) the reverse-microemulsion method, (b) the Stöber method, and (c) the seed-growth method as well as the fluorescence spectrum of QDs (d). The concentration of the DMIP@QDs prepared by the three methods were all $60 \mathrm{mg} / \mathrm{L}$.

week may well be because the CdTe QDs were well protected by the silica shell of the MIPs.

It is well known that fluorescamine itself and its hydrolyzates have no significant fluorescence signal; however, when it is reacted with a compound containing a primary amino group, a compound with a maximum emission wavelength at $475 \mathrm{~nm}$ and strong fluorescence will be generated. Therefore, fluorescamine was employed to investigate the existence of amino groups on the surface of the DMIP@QD particles. As can be seen from Figure 3C, after DMIP@QDs were incubated with fluorescamine for $30 \mathrm{~min}$, a peak of around $475 \mathrm{~nm}$ appeared, which indicated that APTES was introduced to the surface of QDs. All of the results of the SEM, TEM, and fluorescence spectra confirmed that the MIPs were successfully capped on the QDs.

Possible Sensing Mechanism of the DMIP@QDs. The recognition process for TNT by the prepared DMIP@QDs was schematically shown in Figure 1B. As seen, the fluorescence quenching could be attributed to the electron transfer between APTES and TNT. Previous work has shown that a strong charge-transfer interaction occurs between the electrondeficient aromatic ring of TNT and the electron-rich amino group of APTES. ${ }^{25}$ The electron transfer from the amino groups to the aromatic rings leads to the formation of a Meisenheimer complex between TNT and the primary amino groups. Therefore, APTES was used as the functional monomer to recognize TNT. In the mixed solution, the amino groups in the functional molecule of APTES could interact with TNT to form a complex through hydrogen bonding (Figure 4A, inset). This interaction can be confirmed by the UV-vis spectra (Figure 4A). When adding APTES into a TNT solution, a new visible absorbance at $525 \mathrm{~nm}$ could be observed, and the color of the solution changed from colorless into light red (Figure $4 \mathrm{~A}$, inset). The quenching mechanism could be more clearly illustrated in Figure $4 \mathrm{~B}$ as follows. With the existence and increase of TNT, a Meisenheimer complex could be formed between TNT and the primary amino groups on the surface of the QDs. Moreover, it is known that the excitation wavelength for the CdTe QDs used in the present work is $450-580 \mathrm{~nm}$ (Figure 3A) and the absorption wavelength for the Meisenheimer complex is $350-650 \mathrm{~nm}$ (Figure $4 \mathrm{~A}$ ); therefore, there was an excellent overlap for the two spectra, which could very possibly lead to fluorescence resonance energy transfer. The energy of the QDs was transferred to the Meisenheimer complex, which would result in the fluorescence quenching of the QDs. Hence, TNT could be sensed fluorescently.

Analytical Sensitivity of the Sensor. On the basis of the above results, the sensor could be employed for the quantitative determination of TNT. Variables such as the dispersion medium, response time, and amount of DMIP@QDs, which may affect the fluorescence-quenching results, were studied and optimized. During those experiments, the TNT concentration was fixed, and a quenching amount, defined as $\left(F_{0}-F\right) / F_{0}$, was used as the index of quenching capacity. The dispersion medium was first studied. DMIP@QDs dispersed in water had a higher fluorescence intensity but low quenching amount. When water/ethanol was used as the dispersion medium, the fluorescence intensity decreased with the increase of ethanol, whereas the quenching amount increased (Figure S4). Therefore, ethanol was selected as the dispersion medium. The amounts of DMIP@QDs had an obvious effect on the quenching efficency. Too high of an amount of DMIP@QDs would result in too low of a sensitivity, whereas too low of an amount of DMIP@QDs would lead to a narrowing of the linear 

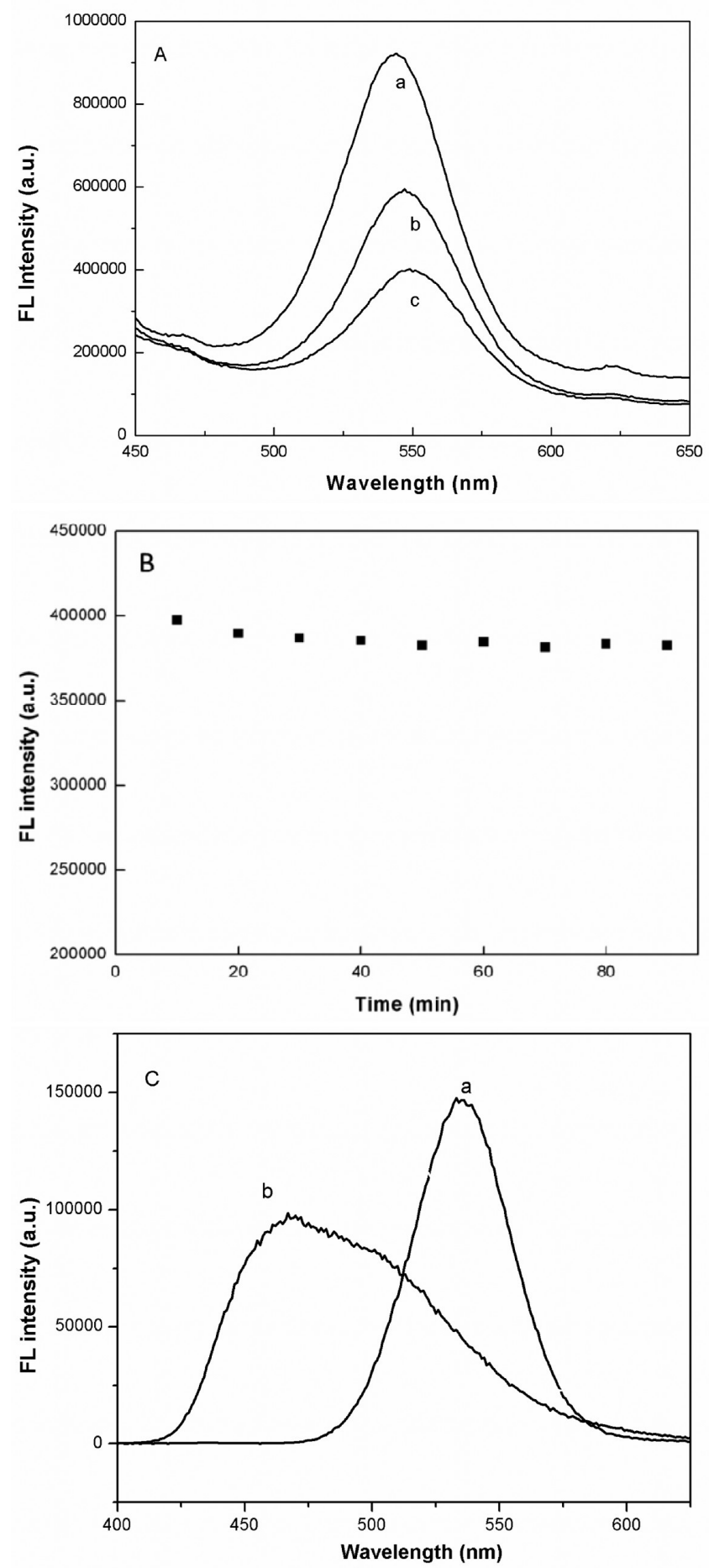

Figure 3. (A) Fluorescence spectra of (a) QDs, (b) DMIP@QDs, and (c) NIP@QDs. (B) Fluorescence intensity change of DMIP@QDs within 90 min. (C) Fluorescence spectra of (a) DMIP@QDs and (b) DMIP@QDs interacting with fluorescamine. The experimental conditions were MIPs or NIPs, $50 \mathrm{mg} / \mathrm{L}$; excited light, $380 \mathrm{~nm}$; silt widths of excitation and emission, 6 and $6 \mathrm{~nm}$, respectively.

range. By changing the amount of DMIP@QDs from 10 to 70 $\mathrm{mg} / \mathrm{L}$, the highest quenching amount appeared at $50 \mathrm{mg} / \mathrm{L}$ (Figure S5). Therefore, the concentration of DMIP@QDs was fixed at $50 \mathrm{mg} / \mathrm{L}$ throughout the work. The response time of the fluorescence intensity for TNT was also studied. From Figure 5A, we can see that when the TNT concentration was fixed the fluorescence intensity decreased rapidly with increasing time in the initial $10 \mathrm{~min}$, after which the curve became flat. Therefore, the sensor had a rapid response speed for TNT, and $10 \mathrm{~min}$ was selected as the response time for the following experiments.

Under the optimal conditions, the fluorescence intensity change with TNT concentration was investigated. It can be seen that the fluorescence intensity of DMIP@QDs was quenched gradually with the increasing concentration of TNT. Generally, the fluorescence quenching depends on the adsorptive affinity of the particles and analytes. In the case of DMIP@QDs, the fluorescence quenching was mainly achieved by the affinity of the imprinted cavities for the template because of the specific interactions. A comparison of Figure 5B,C clearly shows that the decrease of fluorescence intensity of the DMIP@QDs was much larger than that of NIP@QDs, which indicated that the DMIP@QDs could greatly enhance the quenching efficiency, enlarging the spectral sensitivity of DMIP@QDs to TNT.

To evaluate further the analytical performance of the DMIP@QDs, the detection limit and linear range were examined. The linear range was $0.8-30 \mu \mathrm{M}$, with a correlation coefficient of 0.9956 for TNT (Figure 5B, inset). The detection limit, which was calculated as the concentration of TNT for which the quenched amount was three times the standard deviation of the blank signal, of $0.28 \mu \mathrm{M}$ was attained. The precision for the five-replicate detection of TNT was $3.56 \%$ (relative standard deviation, RSD). The sensor was demonstrated to be highly sensitive and highly reliable for the detection of TNT.

Moreover, reusability is also an important index for a novel fluorescent sensor, which is very likely to be a key factor in improving the economic efficiency. The removal-rebinding cycle was repeated five times using the same DMIP@QDs. During the process, the DMIP@QDs could be easily recovered by centrifuging and washing to remove the rebinding TNT with an ethanol/ACN solvent. It was found that the sensor could retain its fluorescence intensity and detection sensitivity during those five recycles, presenting relative standard errors within 1.7\% (Figure S6). The results demonstrated that the DMIP@ QDs could be repeatedly used at least five times without a significant decrease in their quenching capacity for TNT determination.

Molecular Selectivity and Sensing Specificity of the Sensor. The selectivity of DMIP@QDs was further demonstrated by a competitive binding test. To investigate the competitive recognition ability of DMIP@QDs, TNP, DNP, 4$\mathrm{NP}$, phenol, and DNT were used as analogues. As seen from Figure 6, the fluorescence quenching amount was the highest for TNP among these molecules. This phenomenon can be explained as follows: in the synthesis process, many specific recognition sites with respect to the template were generated on the surface of DMIP@QDs, so the template TNP could be bound strongly to the particles and cause significant changes in the fluorescence intensity. Interestingly, the fluorescence quenching amount for TNT was also high and only slightly lower than that for TNP, considering that TNT has an extremly similar shape and volume to TNP (Figures 1A and S1). That the DMIP@QDs showed excellent recognition to TNT indicated TNP is an ideal choice as a dummy template for TNT.

However, as shown in Figure 6, the competitive molecules including DNP, 4-NP, phenol, and DNT caused little 
(A)

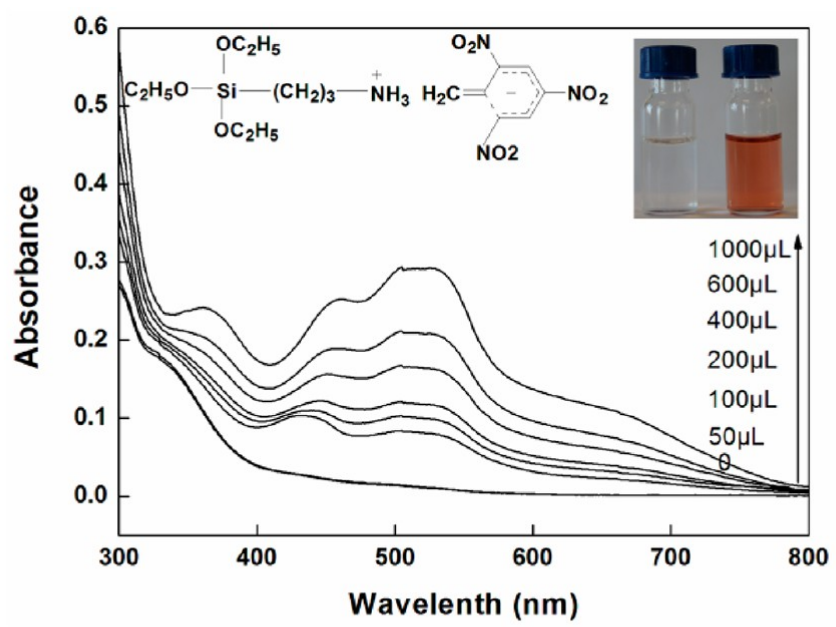

(B)

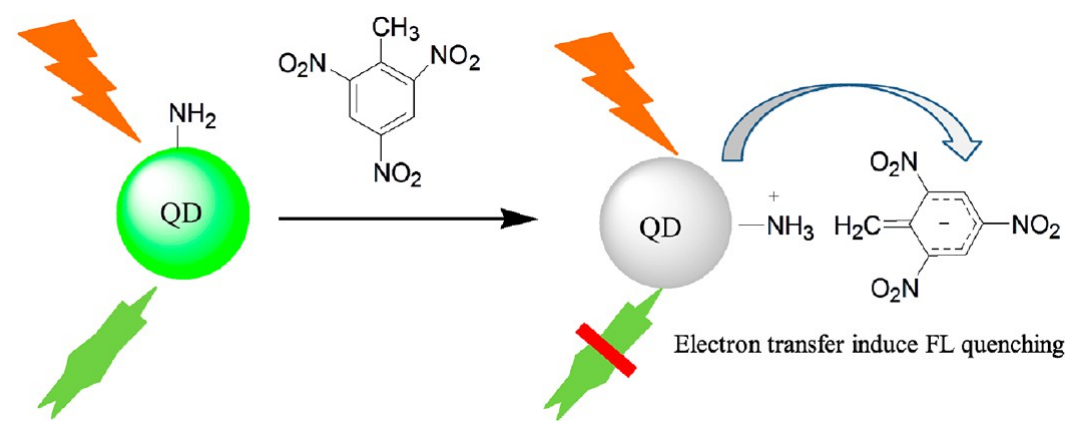

Figure 4. (A) UV-vis spectra for the interaction of TNT with APTES. The inset shows the schematic illustration for the charge-transfer complex interactions between APTES and TNT and the corresponding color for the interaction of TNT and APTES. (B) Schematic for the QDs quenching mechanism on the basis of the electron-transfer-induced resonance energy transfer.

fluorescence quenching when compared to that of TNT and TNP. This result can be reasonably interpreted by the following two aspects. First, DNP, 4-NP, phenol, and DNT have a much different shape and volume from TNP. Therefore, although they also could interact with APTES by hydrogen bonding, the recognition sites of the imprinting cavities were not complementary to them, resulting in less chance to quench the fluorescence of the QDs. Second, with fewer nitro groups in the molecules, the electron-withdrawing ability of DNP, 4-NP, phenol, and DNT were weaker, resulting in a fluorescence quenching ability that was much lower than that of TNP.

Moreover, as a comparison, the NIP@QDs were also prepared for fluorometry. It was observed from Figure 6 that there was a quite close and very little fluorescence quenching of all the analogues and TNT for the NIPs, suggesting that the NIP@QDs could not effectively bind TNT as well as its analogues. It was also found that the amino groups provided binding sites on the surface of MIPs- or NIPs-capped QDs. Therefore, both MIPs and NIPs had responses to TNT, but the quencher constants were significantly different. The fluorescence quenching in this system followed the Stern-Volmer equation $^{38-40}$

$$
F_{0} / F=1+K_{\mathrm{sv}} C_{\mathrm{q}}
$$

where $F_{0}$ and $F$ are the fluorescence intensity in the absence and presence of quencher, respectively, $C_{\mathrm{q}}$ is the concentration of the quencher, and $K_{\mathrm{sv}}$ is the quenching constant for the quencher. Generally, fluorescence quenching includes two kinds of quenching, dynamic quenching and static quenching. ${ }^{38,39}$ Temperature-varying experiments, absorption spectrum measurements, and fluorescence lifetime measurements are often used to identify the type of quenching and its mechanism. ${ }^{38,39}$ Considering the available experimental conditions of our lab, the absorption spectrum of DMIP@QDs was tested to check the quenching mechanism. It was observed that the absorption spectra of DMIP@QDs changed with the addition of the quencher, from which static quenching could be deduced. ${ }^{38,39}$

However, it is well known that the imprinting factor $(\alpha)$ is an important index to evaluate the selectivity of the imprinted materials, which is generally defined as $\alpha=Q_{\text {MIPs }} / Q_{\text {NIPs, }}$, where $Q_{\text {MIPs }}$ and $Q_{\text {NIPs }}$ are adsorption capacity of the template or the analogues on MIPs and NIPs, respectively. Herein, the imprinting factor can be calculated as the ratio of the $K_{\mathrm{sv}}$ value of the MIPs and NIPs. Under optimum conditions, the imprinting factor was 1.785 (calculated from Figure 5B,C), indicating that the MIPs had a much better selectivity than the NIPs. Therefore, the results shown in Figure 6 also proved that the DMIP@QDs possessed high molecular selectivity and high sensing specificity.

Practical Application and Performance Comparison. The sensing of TNT in soil samples was carried out to investigate further the practical application of this method. 

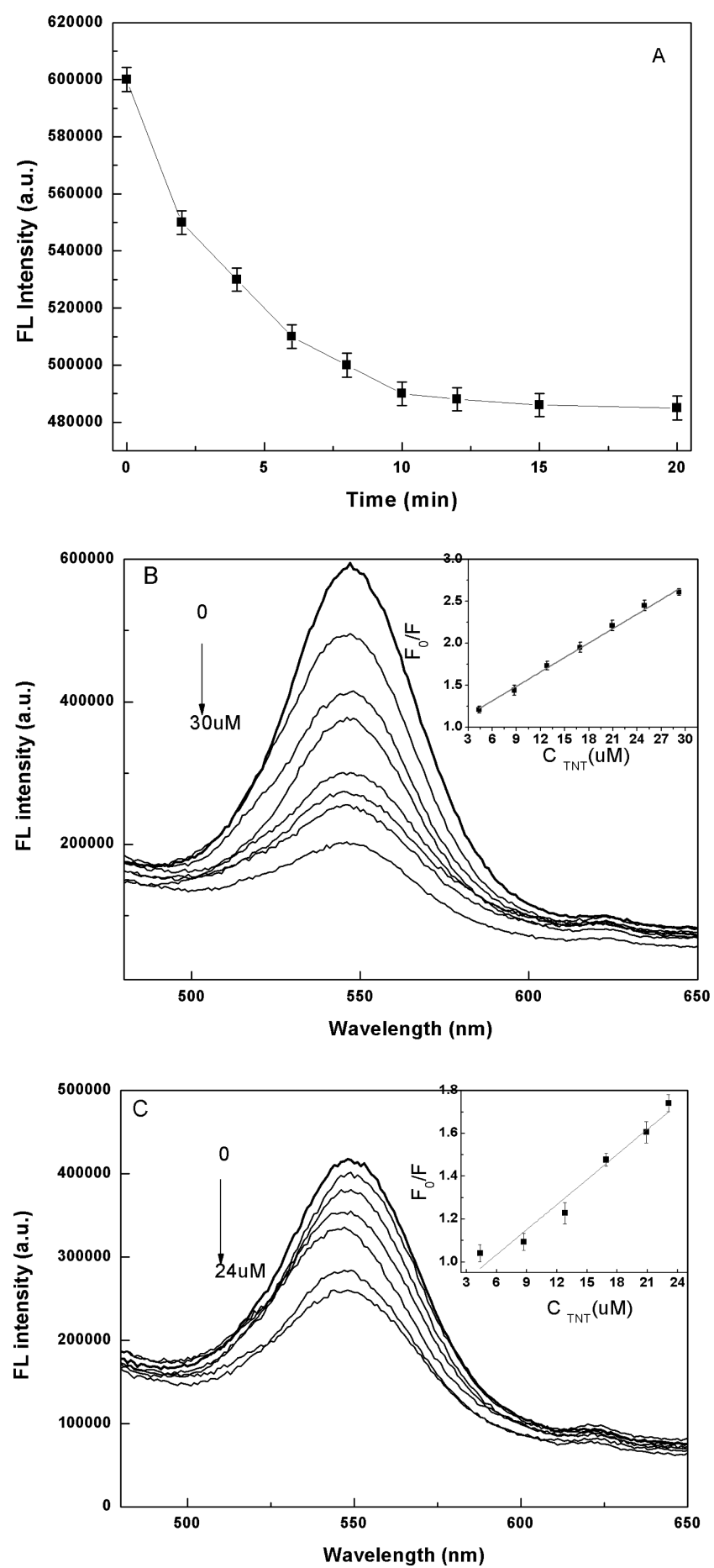

Figure 5. (A) Fluorescence response time of DMIP@QDs for TNT. (B, C) Fluorescence emission spectra of MIPs-capped (B) and NIPscapped (C) QDs $(50 \mathrm{mg} / \mathrm{L})$ with an increasing TNT concentration in an ethanol solution. The inset graphs show the Stern-Volmer plots for MIPs-capped QDs and NIPs-capped QDs. The experimental conditions were MIPs or NIPs, $50 \mathrm{mg} / \mathrm{L}$; excited light, $380 \mathrm{~nm}$; silt widths of excitation and emission, 6 and $6 \mathrm{~nm}$, respectively.

TNT standard solutions were tested side by side with the unknown counterparts, focusing on the linear range of the fluorescence intensity changes versus concentration, permitting the construction of a standard curve. No response of the solutions was observed until the samples were spiked with TNT. Next, TNT-spiked experiments were performed, and the

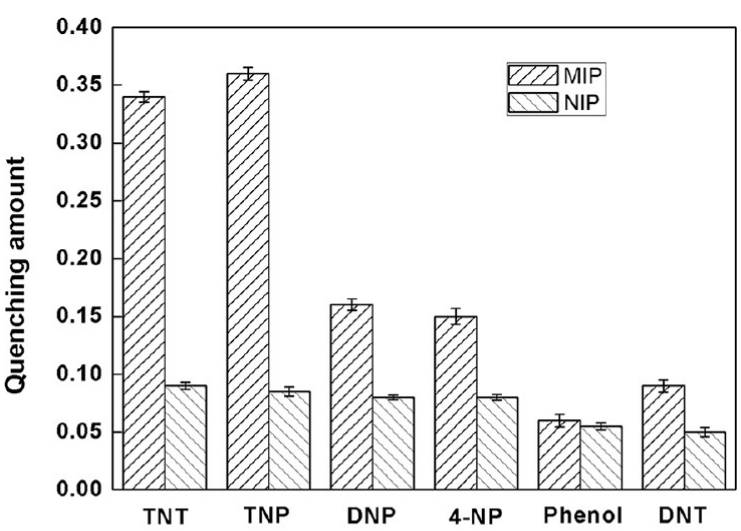<smiles>Cc1c([N+](=O)[O-])cc([N+](=O)[O-])cc1[N+](=O)[O-]</smiles>

TNT<smiles>O=[N+]([O-])c1cc([N+](=O)[O-])c(O)c([N+](=O)[O-])c1</smiles>

TNP<smiles>O=[N+]([O-])c1ccc(O)c([N+](=O)[O-])c1</smiles>

DNP<smiles>O=[N+]([O-])c1ccc(O)cc1</smiles>

4-NP<smiles>Oc1ccccc1</smiles>

Phenol<smiles>Cc1ccc([N+](=O)[O-])cc1[N+](=O)[O-]</smiles>

DNT
Figure 6. Quenching amounts of DMIP@QDs for TNT and its structural analogues. The quenching amount was defined as $\left(F_{0}-F\right) /$ $F_{0}$. The experimental conditions were MIPs or NIPs, $50 \mathrm{mg} / \mathrm{L} ; C_{0}, 10$ $\mu \mathrm{M}$; excited light, $380 \mathrm{~nm}$; silt widths of excitation and emission, 6 and $6 \mathrm{~nm}$, respectively.

recoveries reached $90.3 \%$ or higher (Table 1 ). The values determined by the DMIP@QDs sensor followed the same

Table 1. Spiked Recoveries and Relative Standard Deviations (RSD, \%, $n=5$ ) in Soil Samples Using DMIP@QDs and HPLC Analysis

\begin{tabular}{ccccccc} 
& & \multicolumn{2}{c}{ DMIP@QDs } & & \multicolumn{2}{c}{ HPLC } \\
\cline { 3 - 4 } \cline { 6 - 7 } sample & added $(\mu \mathrm{M})$ & recovery (\%) & RSD (\%) & & recovery (\%) & RSD (\%) \\
soil & 1 & 93.9 & 4.53 & & 98.5 & 2.12 \\
& 5 & 97.8 & 3.67 & & 108 & 1.96 \\
& 10 & 90.3 & 5.12 & & 103 & 1.56 \\
\hline
\end{tabular}

trend as those found by HPLC (Table 1). This sensing method has promising potential for use in environmental applications for TNT detection.

Much excellent work about chemosensors for TNT detection has been reported and some were summarized and compared with this present work, with the results were listed in Table 2 . In comparison with the other work on MIP@QDs for use in detection applications, ${ }^{25-28,41,42}$ the developed DMIP@QDs showed excellent selectivity but low sensitivity. This problem of low sensitivity commonly exists for MIP@QDs-based systems. For example, Zhang et al. ${ }^{22}$ prepared a sensor for cytochrome c on the basis of MIP-coated CdTe QDs. The linear range for cytochrome $\mathrm{c}$ was from 0.97 to $24 \mu \mathrm{M}$, and the LOD was 0.41 $\mu \mathrm{M}$. Lin et al. ${ }^{20}$ reported a chemiluminescence system for the 
Table 2. Comparisons of the Analytical Performances with Other Reported Methods for TNT Sensing

\begin{tabular}{|c|c|c|c|c|c|c|}
\hline sensor system & linear range & mechanism & response mode & selectivity $^{a}$ & limit of detection & ref \\
\hline amine-capped QDs & $10-100 \mu \mathrm{M}$ & & & moderate & $1 \mathrm{nM}$ & 25 \\
\hline Au nanorod-QDs & $1.1-66 \mathrm{nM}$ & FRET $^{b}$ & $\mathrm{FL}^{c}$ turn on & excellent & $0.10 \mathrm{nM}$ & 26 \\
\hline dye-modified silica nanoparticles & $10-100 \mu \mathrm{M}$ & FRET & FL quenching & moderate & $1 \mathrm{nM}$ & 27 \\
\hline Mn dopped $\mathrm{ZnS}$ QDs & $2.5-450 \mathrm{nM}$ & $\mathrm{RTP}^{d}$ and $\mathrm{RS}^{e}$ & RTP quenching RS enhancement & moderate & $0.8 \mathrm{nM}$ & 28 \\
\hline fluorescence-labeled MIPs & & & FL quenching & excellent & $40.7 \mu \mathrm{M}$ & 29 \\
\hline dual-emission CdTe QDs & & FRET & FL quenching & moderate & $5 \mathrm{ng} / \mathrm{mm}^{2}$ & 36 \\
\hline QDs-antibody fragment & & FRET & recovery & moderate & & 37 \\
\hline MIP-capped CdTe QDs & $0.8-30 \mu \mathrm{M}$ & electron transfer & FL quenching & excellent & $0.28 \mu \mathrm{M}$ & this work \\
\hline
\end{tabular}

${ }^{a}$ Moderate $=$ cannot distinguish TNT from its analogues such as nitrobenzene and DNT. Excellent $=$ can distinguish TNT from its analogues. ${ }^{b}$ Fluorescence resonance energy transfer. ${ }^{c}$ Fluorescence. ${ }^{d}$ Room temperature phosphorescence. ${ }^{e}$ Rayleigh scattering.

detection of 4-NP on the basis of MIP-capped Mn-doped $\mathrm{ZnS}$ QDs. The linear range was from 0.1 to $40 \mu \mathrm{M}$, and the LOD was $76 \mathrm{nM}$. Lee and his group ${ }^{43}$ prepared QDs-incorporated MIPs for proteins. The LODs of creatinine, albumin, and lysozyme were $0.635,0.000898$, and $0.00021 \mathrm{mg} / \mathrm{mL}$, respectively. This can be attributed to the inherent property of MIPs. During the preparation process for the MIPs, more cross linker was used to obtain highly rigid recognition sites; therefore, the interaction sites between the target molecule and the functional monomer were relatively small, which reduced the sensitivity of the sensor. This problem has been commonly present in the field of MIPs, and much work still needs to be done to improve the sensitivity of MIP@QDs sensors while retaining the high selectivity. For example, reducing the shell thickness is one effective way. In view of the relationship between the quenching efficiency and the MIP shell thickness, an ultrathin shell will be the ideal goal of MIP@QDs sensors for higher sensitivity and shorter analysis time. Excitingly, by comparing the MIP@QDs with fluorescence-labeled MIPs, ${ }^{30}$ the sensitivity and selectivity of the present method had been greatly enhanced. As a new material, our developed DMIP@ QDs fluorescent sensor proved to be an ideal candidate for the selective and sensitive detection of TNT.

\section{CONCLUSIONS}

A novel DMIP@QDs sensor was developed through a sol-gel seed-growth method by virtue of a dummy molecularimprinting technique for the detection of TNT on the basis of an electron-transfer-induced fluorescence quenching mechanism.The DMIP@QDs integrated the high selectivity of MIPs and the strong fluorescence property of QDs, demonstrating a highly selective and sensitive recognition and determination of TNT. The simple, rapid, and reliable DMIP@QDs sensing strategy opens up attractive perspectives for TNT monitoring. More efforts still need to be made to improve further the sensitivity of MIP@QDs-based systems while retaining their high selectivity.

\section{ASSOCIATED CONTENT}

\section{(S Supporting Information}

Bond lengths and bond angles of TNT and TNP, fluorescence emission spectra of DMIP@QD, fluorescence intensity changes in DMIP@QDs within 7 days, effect of the dispersion medium on the quenching efficiency, effect of the amount of DMIP@ QDs on the quenching efficiency, and recovery of the FL intensity of DMIP@QDs after the removal of TNT. This material is available free of charge via the Internet at http:// pubs.acs.org.

\section{AUTHOR INFORMATION}

\section{Corresponding Author}

*E-mail: lxchen@yic.ac.cn. Fax/Tel: +86-535 2109130.

\section{Notes}

The authors declare no competing financial interest.

\section{ACKNOWLEDGMENTS}

This work was financially supported by the Scientific Research Foundation for the Returned Overseas Chinese Scholars, the State Education Ministry, the National Natural Science Foundation of China (21105117, 21275158, and 21275068), the Scholarship Award for Excellent Doctoral Student granted by the Ministry of Education of China, and the 100 Talents Program of the Chinese Academy of Sciences.

\section{REFERENCES}

(1) Ali, E.; Zheng, Y.; Yu, H.; Ying, J. Anal. Chem. 2007, 79, 94529458.

(2) Dong, H.; Gao, W.; Yan, F.; Ji, H.; Ju, H. Anal. Chem. 2010, 82, 5511-5517.

(3) Xu, X.; Liu, X.; Nie, Z.; Pan, Y.; Guo, M.; Yao, S. Anal. Chem. 2011, 83, 52-59.

(4) Wang, Y. Q.; Chen, L. X. J. Nanomed. Nanotechnol. 2011, 7, 385402 .

(5) Liu, M.; Xu, L.; Cheng, W.; Zeng, Y.; Yan, Z. Spectrochim. Acta, Part A 2008, 70, 1198-1202.

(6) Chen, L. X.; Xu, S. F.; Li, J. H. Chem. Soc. Rev. 2011, 40, 29222942.

(7) Song, X. L.; Li, J. H.; Wang, J. T.; Chen, L. X. Talanta 2009, 80, 694-702.

(8) Li, Y.; Ding, M. J.; Wang, S.; Wang, R. Y.; Wu, X. L.; Wen, T. T.; Yuan, L. H.; Dai, P.; Lin, Y. H.; Zhou, X. M. ACS Appl. Mater. Interfaces 2011, 3, 3308-3315.

(9) Xu, S. F.; Chen, L. X.; Li, J. H.; Qin, W.; Ma, J. J. Mater. Chem. 2011, 21, 12047-12053.

(10) Song, X. L.; Li, J. H.; Xu, S. F.; Ying, R.; Ma, J.; Liao, C.; Liu, D.; Yu, J.; Chen, L. X. Talanta 2012, 99, 75-82.

(11) Xu, S. F.; Li, J. H.; Chen, L. X. Talanta 2011, 85, 282-289.

(12) Lee, M. H.; Thomas, J. L.; Chen, Y. C.; Wang, H. Y.; Lin, H. Y. ACS Appl. Mater. Interfaces 2012, 4, 916-921.

(13) Xu, S. F.; Li, J. H.; Chen, L. X. J. Mater. Chem. 2011, 21, 43464351.

(14) Xu, S. F.; Chen, L. X.; Li, J. H.; Guan, Y. F.; Lu, H. Z. J. Hazard. Mater. 2012, 237-238, 347-354.

(15) Li, J. H.; Wen, Y. Y.; Chen, L. X. Chin. J. Chromatogr. 2013, 31, $181-184$.

(16) Lee, M. H.; Thomas, J. L.; Ho, M. H.; Yuan, C.; Lin, H. Y. ACS Appl. Mater. Interfaces 2010, 2, 1729-1736.

(17) Li, J. H.; Zhang, Z.; Xu, S. F.; Xiong, H.; Peng, H.; Zhou, N.; Chen, L. X. J. Mater. Chem. 2011, 21, 19267-19274.

(18) Xu, S. F.; Li, J. H.; Song, X.; Liu, J.; Lu, H. Z.; Chen, L. X. Anal. Methods 2013, 5, 124-133. 
(19) Wang, H.; He, Y.; Ji, T.; Yan, X. Anal. Chem. 2009, 81, 16151621.

(20) Liu, J.; Chen, H.; Lin, Z.; Lin, J. Anal. Chem. 2010, 82, 73807386.

(21) Li, H.; Li, Y.; Cheng, J. Chem. Mater. 2010, 22, 2451-2457.

(22) Zhang, W.; He, X.; Chen, Y.; Li, W.; Zhang, Y. Biosens. Bioelectron. 2011, 26, 2553-2558.

(23) Lee, M.; Chen, Y.; Ho, M.; Lin, H. Anal. Bioanal. Chem. 2010, 397, 1457-1466.

(24) Dan, L.; Wang, H. F. Anal. Chem. 2013, 85, 4844-4848.

(25) Woodka, M.; Schnee, V. P. Anal. Chem. 2010, 82, 9917-9924.

(26) Tu, R.; Liu, B.; Wang, Z.; Gao, D.; Wang, F.; Fang, Q.; Zhang, Z. Anal. Chem. 2008, 80, 3458-3465.

(27) Xia, Y.; Song, L.; Zhu, C. Anal. Chem. 2011, 83, 1401-1407.

(28) Gao, D.; Wang, Z.; Liu, B.; Ni, L.; Wu, M.; Zhang, Z. Anal. Chem. 2008, 80, 8545-8553.

(29) Zou, W.; Sheng, D.; Ge, X.; Qiao, J.; Lian, H. Anal. Chem. 2011, 83, 30-37.

(30) Stringer, R. C.; Gangopadhyay, S.; Grant, S. A. Anal. Chem. 2010, 82, 4015-4019.

(31) Thomas, S. W., III; Joly, G. D.; Swager, T. M. Chem. Rev. 2007, 107, 1339-1386.

(32) Zhao, W. H.; Sheng, N.; Zhu, R.; Wei, F. D.; Cai, Z.; Zhai, M. J.; Du, S. H.; Hu, Q. J. Hazard. Mater. 2010, 179, 223-229.

(33) Chen, Y. P.; Wang, D. N.; Yin, Y. M.; Wang, L. Y.; Wang, X. F.; Xie, M. X. J. Agric. Food Chem. 2012, 60, 10472-10479.

(34) Wang, C.; Ma, Q.; Dou, W.; Kanwal, S.; Wang, G.; Yuan, P.; Su, X. Talanta 2009, 77, 1358-1364.

(35) Nann, T.; Mulvaney, P. Angew. Chem., Int. Ed. 2004, 43, 53935396.

(36) Goldman, E. R.; Cohill, T. J.; Patterson, C. H.; Anderson, G. P.; Kusterbeck, A. W.; Mauro, J. M. Environ. Sci. Technol. 2003, 37, 47334736.

(37) Wang, L. J. Phys. Chem. B 2001, 105, 2360-2364.

(38) Kamat, B. P.; Seetharamappa, J. J. Chem. Sci. 2005, 117, 649655.

(39) Cheng, P. P. H.; Silvester, D.; Wang, G.; Kalyuzhny, G.; Douglas, A.; Murray, R. W. J. Phys. Chem. B 2006, 110, 4637-4644.

(40) Gopal, R.; Lee, J. K.; Lee, J. H.; Kim, Y. G.; Oh, G. C.; Seo, C. H.; Park, Y. Int. J. Mol. Sci. 2013, 14, 2190-2202.

(41) Zhang, K.; Zhou, H.; Mei, Q.; Wang, S.; Guan, G.; Liu, R.; Zhang, J.; Zhang, Z. J. Am. Chem. Soc. 2011, 133, 8424-8427.

(42) Goldman, E. R.; Medinta, I. L.; Whitley, J. L.; Hayhurst, A.; Clapp, A. R.; Uyeda, H. T.; Deschamps, J. R.; Lassman, M. E.; Mattoussi, H. J. Am. Chem. Soc. 2005, 127, 6744-6751.

(43) Lin, H.; Ho, M.; Lee, M. Biosens. Bioelectron. 2009, 25, 579-586. 